\title{
Towards Self Validation: Progress and Roadmap for Automating the Validation of BioSense Partner Facilities
}

\author{
Travis Mayo ${ }^{1}$, Matthew Dollacker ${ }^{1}$, Corey Cooper ${ }^{1}$ and Sara Imholte ${ }^{2}$ \\ ${ }^{1} \mathrm{CDC}$ Contractor, InductiveHealth Informatics, Atlanta, GA, USA; ${ }^{2}$ Arizona Department of Health Services, Phoenix, AZ, USA
}

\section{Objective}

This session will inform the BioSense Community about data validation advancements implemented this past year as well as future plans to improve the BioSense validation process to achieve emergency department representativeness goals.

\section{Introduction}

One of the greatest hurdles for BioSense Onboarding is the process of validating data received to ensure it contains Data Elements of Interest (DEOI) needed for syndromic surveillance. Efforts to automate this process are critical to meet existing and future demands for facility onboarding requests as well as provide a foundation for data quality assurance efforts. By automating the validation process, BioSense hopes to:

1. Reduce costs associated with the iterative validation process.

2. Improve BioSense response times for assistance with onboarding.

3. Improve documentation to partners about requirements and communicate changes to DEOI.

4. Provide a better foundation for data quality initiatives.

Efforts to improve data validation are being developed in alignment with BioSense future initiatives and will apply to both BioSense, Essence and other BioSense program applications.

BioSense Onboarding identified critical success factors by participating in ISDS workgroup initiatives for Onboarding and Data Quality and soliciting feedback from key jurisdictional partners. These critical success factors include; improved documentation, access to raw data, and faster validation response time.

\section{Description}

Panelists will review the critical success factors and discuss the improvements and advancements made to the onboarding validation process.

Issues discussed will include:

- Documentation

- Data Access

- Validation Response Time

Documentation improvements include the release of the first BioSense Onboarding Implementation Guide and a new BioSense Data Validation Compliance Report and Facility Approval Tracking Report.

Data access is important to Jurisdiction Administrators so that they can perform initial quality assurance during onboarding, assess continuous facility reporting, and perform continuous data quality initiatives. BioSense Onboarding has advanced this initiative by working to provide jurisdiction administrators with direct access to raw data within their jurisdictional SFTP folders as well as providing access to Stage_1 database tables which hold the raw form of preprocessed DEOI data values that are extracted from the HL7 file.

Validation Response Time improvements include a new helpdesk portal to better track requests and ensure quick response times. Additionally, the newly developed Data Validation Compliance Report provides jurisdiction administrators and developers with better feedback about DEOI compliance rates to enable faster issue resolution. Further improvements are being planned to include sets of automated SQL validation queries, validation description information, as well as proposed plans to create a portal to perform self-validation utilizing these tools thereby providing immediate validation feedback.

\section{Audience Engagement}

Audience participation will focus on discussion of the continued improvements to BioSense Data Validation process and how they impact jurisdictional onboarding initiatives. Feedback will be used to help facilitate the continuous improvement initiatives for the BioSense Onboarding Community.

Keywords

NSSP; Onboarding; Validation; Automation

\section{References}

1. http://www.cdc.gov/phin/phinGuides

2. BioSense Onboarding Implementation Guide

3. BioSense Data Validation Compliance Report

4. BioSense Facility Approval Tracking Report 